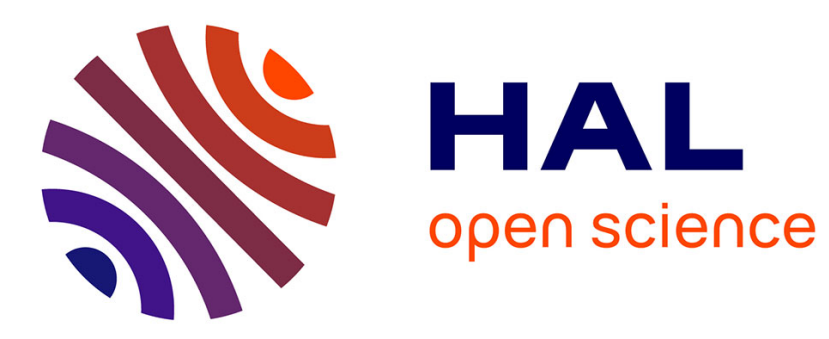

\title{
An Adaptive Sliding-Mode Observer for a Class of Uncertain Nonlinear Systems
}

Héctor Ríos, Denis Efimov, Wilfrid Perruquetti

\section{To cite this version:}

Héctor Ríos, Denis Efimov, Wilfrid Perruquetti. An Adaptive Sliding-Mode Observer for a Class of Uncertain Nonlinear Systems. International Journal of Adaptive Control and Signal Processing, 2018, 32 (3), pp.511 - 527. 10.1002/acs.2857 . hal-01889143

\section{HAL Id: hal-01889143 \\ https://hal.inria.fr/hal-01889143}

Submitted on 5 Oct 2018

HAL is a multi-disciplinary open access archive for the deposit and dissemination of scientific research documents, whether they are published or not. The documents may come from teaching and research institutions in France or abroad, or from public or private research centers.
L'archive ouverte pluridisciplinaire HAL, est destinée au dépôt et à la diffusion de documents scientifiques de niveau recherche, publiés ou non, émanant des établissements d'enseignement et de recherche français ou étrangers, des laboratoires publics ou privés. 


\title{
An Adaptive Sliding-Mode Observer for a Class of Uncertain Nonlinear Systems
}

\author{
H. Ríos ${ }^{1 *}$, D. Efimov ${ }^{2,3}$ and W. Perruquetti ${ }^{2}$ \\ ${ }^{1}$ CONACYT - TECNM/Instituto Tecnológico de La Laguna, División de Estudios de Posgrado e Investigación, Blvd. \\ Revolución y Cuauhtémoc S/N, C.P. 27000, Torreón, Coahuila, México. \\ ${ }^{2}$ Non-A team @ Inria, Parc Scientifique de la Haute Borne, 40 avenue Halley, 59650 Villeneuve d'Ascq, France and \\ CRIStAL (UMR-CNRS 9189), Ecole Centrale de Lille, BP 48, Cité Scientifique, 59651 Villeneuve-d'Ascq, France. \\ ${ }^{3}$ Department of Control Systems and Informatics, Saint Petersburg State University of Information Technologies \\ Mechanics and Optics (ITMO), Kronverkskiy av. 49, Saint Petersburg, 197101, Russia.
}

\begin{abstract}
SUMMARY
In this paper the problem of simultaneous state and parameter estimation is studied for a class of uncertain nonlinear systems. A nonlinear adaptive sliding-mode observer is proposed based on a nonlinear parameter estimation algorithm. It is shown that such a nonlinear algorithm provides a rate of convergence faster than exponential, i.e. faster than the classic linear algorithm. Then, the proposed parameter estimation algorithm is included in the structure of a sliding-mode state observer providing an ultimate bound for the full estimation error attenuating the effects of the external disturbances. Moreover, the synthesis of the observer is given in terms of linear matrix inequalities. The corresponding proofs of convergence are developed based on Lyapunov function approach and input-to-state stability theory. Some simulation results illustrate the efficiency of the proposed adaptive sliding-mode observer.
\end{abstract}

Received ..

KEY WORDS: Adaptive observer, Sliding-modes, Nonlinear systems.

\section{INTRODUCTION}

Despite a significant advance in development of identification tools for nonlinear systems, the controllers have to cope with a high level of uncertainty, mainly due to lack of knowledge on the system parameters and the fact that the whole system state is not available to measure. This situation justifies the need for the adaptive control design that has received a great deal of attention in control theory during the last decades. Due to this attention, this area has grown to turn into one of the widest in terms of algorithms, techniques for design, analytical tools, and so on (see, for instance [1] and [2]). One important problem in the adaptive control area is the design of adaptive observers, i.e. the design of observers estimating simultaneously the whole state and the parameters of the system by some on-line adaptation law [3].

In this context, there exist a lot of literature related to the adaptive observers design for linear systems (see, for instance [4], [5], [6], and [7]). Most of these results are based on appropriated change of state coordinates to some canonical form in order to provide a state estimation together with persistence of excitation conditions to ensure the parameter estimation.

*Correspondence to: H. Ríos, Instituto Tecnológico de La Laguna, División de Estudios de Posgrado e Investigación, Blvd. Revolución y Cuauhtémoc S/N, C.P. 27000, Torreón, Coahuila, México. Email: hriosbecorreo.itlalaguna.edu.mx 
For nonlinear systems, one of the first results was proposed by [8] extending some linear results. In the same vein, several results based on output injection transformations are given for nonlinear systems that are equivalent to linear observable systems in the Brunovsky observer form (see, for example [9] and [10]). Afterwards, in [11] a unifying adaptive observer form is proposed for nonlinear systems providing asymptotic state estimation as well as parameter estimation under some passivity-like conditions. For multiple-input multiple-output (MIMO) linear time-varying systems, an adaptive observer is proposed by [12] and it is also valid for affine state nonlinear systems. In [13], a more general design of adaptive observers is proposed for a class of single-output uniformly observable nonlinear systems. For the case of uniformly observable multiple-input multiple-output nonlinear systems, in [14] an adaptive observer is proposed to exponentially estimate the state and the unknown parameters under a persistent excitation condition. The structure of this observer gives some flexibility to obtain high-gain-like observers and adaptive sliding-mode-like observers. In [15] a robust adaptive observer is provided for nonlinear systems with disturbances and unmodeled dynamics based on adaptive nonlinear damping. Nevertheless, such an observer is just able to estimate the state. In [16], a redesign of adaptive observers is proposed for nonlinear systems based on adaptive laws that use delayed measurements. These delayed observers improve the performance of the parameter estimation but increase the computational load. More recently, in [17], based on the concepts of weakly attracting sets and non-uniform convergence, an adaptive observer is proposed to asymptotic reconstruction of the state and parameter values in a particular class of forwardcomplete single input-single-output nonlinear systems. Adaptive impulsive observers are proposed in [18] and [19] for a class of nonlinear systems. Particularly, in [18], the proposed observer is modelled by an impulsive differential equation, the asymptotic convergence is analyzed based on a time-varying Lyapunov function approach and some sufficient conditions are formulated in terms of linear matrix inequalities. On the other hand, in [19], a new impulsive adaptive observer shows that an impulsive feedback can improve the convergence rate or relax the requirement on persistency of excitation. For a class of nonlinear sampled-data systems, in [20] a new hybrid adaptive observer is designed and shown to be exponentially convergent under some common conditions. In the same vein, in [21] an adaptive observer is proposed for systems with cascade structure including finitedimensional dynamics followed by infinite-dimensional dynamics. The given adaptive observer is based on a combination of backstepping method and the extended Kalman observer approach, and it is shown to be exponentially convergent under a persistent excitation condition. However, most of the works previously mentioned do not consider external disturbances, in addition, the convergence rate of the parameter and state estimation errors is exponential or asymptotic. In addition, it has been shown in [19] that an improvement of convergence rate of parameter estimation error cannot be achieved by a simple increasing the observer gains.

Some other solutions have been proposed in the sliding-mode area where the presented solutions demonstrate a remarkable insensitivity with respect to certain class of external disturbances [22]. In the context of fault detection, in [23] an adaptive sliding-mode observer is provided for a class of nonlinear systems with unknown parameters and faults. Using the inherent features of the slidingmode observers, a fault reconstruction is given under relative degree of the output with respect to the fault equal to one. Finally, it is worth saying that most of the mentioned adaptive observers propose linear parameter estimation algorithms.

\subsection{Main Contribution}

This paper contributes with a nonlinear adaptive sliding-mode observer based on a nonlinear parameter identification algorithm for uncertain nonlinear systems. The proposed nonlinear adaptive sliding-mode observer is a modified version of that one proposed in [24]. Such a modification lies in the inclusion of a nonlinear parameter identification algorithm that provides a rate of convergence faster than exponential, i.e. faster than classic linear algorithms. Then, the proposed parameter identification algorithm is included in the structure of a sliding-mode state observer providing an ultimate bound for the state and parameter estimation error attenuating the effects of the external disturbances. This solution ensures that the level of observer robustness with respect to some matched disturbances is improved as well as the rate of convergence. Moreover, the synthesis of the 
observer is given in terms of linear matrix inequalities. The corresponding proofs of convergence are developed based on Lyapunov function approach and input-to-state stability theory. Some simulation results illustrate the efficiency of the proposed nonlinear adaptive sliding-mode observer.

\subsection{Structure of the paper}

The problem statement is presented in the Section 2. The proposed adaptive sliding-mode observer as well as the convergence proof are given in Section 3. The simulation results are illustrated by Section 4. Some concluding remarks are discussed in Section 5. Finally, the proofs of all proposed results are postponed to the Appendix.

\subsection{Notation}

Let $\|q\|$ denote the Euclidean norm of a vector $q \in \mathbb{R}^{n} ; \overline{1, n}$ a sequence of integers $1, \ldots, n$; and $I_{n}$ an identity matrix of dimension $n \times n$. For a matrix $Q \in \mathbb{R}^{m \times n}$, denote its smallest singular value $\sigma_{\min }(Q)=\sqrt{\lambda_{\min }\left(Q^{T} Q\right)}$ and its induced norm as $\|Q\|:=\sqrt{\lambda_{\max }\left(Q^{T} Q\right)}=\sigma_{\max }(Q)$, where $\lambda_{\max }$ (respectively, $\lambda_{\min }$ ) is the maximum (respectively, the minimum) eigenvalue, and $\sigma_{\max }$ is the largest singular value. For a Lebesgue measurable function $u: \mathbb{R}_{\geq 0} \rightarrow \mathbb{R}^{m}$ define the norm $\|u\|_{\left(t_{0}, t_{1}\right)}:=$

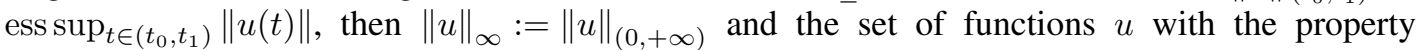
$\|u\|_{\infty}<+\infty$ is denoted as $\mathcal{L}_{\infty}$. For a matrix $Q: \mathbb{R}_{\geq 0} \rightarrow \mathbb{R}^{m \times n}$ denote $\|Q\|_{\infty}:=\|Q\|_{(0,+\infty)}$. A continuous function $\alpha: \mathbb{R}_{\geq 0} \rightarrow \mathbb{R}_{\geq 0}$ belongs to class $\mathcal{K}$ if it is strictly increasing and $\alpha(0)=0$; it belongs to class $\mathcal{K}_{\infty}$ if it is also unbounded. A continuous function $\beta: \mathbb{R}_{\geq 0} \times \mathbb{R}_{\geq 0} \rightarrow \mathbb{R}_{\geq 0}$ belongs to class $\mathcal{K} \mathcal{L}$ if for each fixed $s, \beta(r, s) \in \mathcal{K}$ with respect to $r$, and for each fixed $r, \beta(r, s)$ is decreasing to zero with respect to $s$. The notation $\nabla V(x) f(x)$ denote the directional derivative of a continuously differentiable function $V$ with respect to the vector field $f$ evaluated at any point $x$.

\section{PROBLEM STATEMENT}

Consider a class of uncertain nonlinear systems that can be written, essentially after a change of coordinates, as follows

$$
\begin{aligned}
& \dot{x}=A x+\phi(y, u)+G(t, y, u) \theta+D w, \\
& y=C x,
\end{aligned}
$$

where $x \in \mathbb{R}^{n}$ is the state vector, $y \in \mathbb{R}^{p}$ is the measurable output vector, $u \in \mathbb{R}^{m}$ is the control input vector, $\theta \in \mathbb{R}^{q}$ is a vector of unknown constant parameters, and $w \in \mathbb{R}^{l}$ is a vector of external disturbances. The matrices $A, C$ and $D$ are known, they have corresponding dimensions, and the pair $(A, C)$ is detectable. The functions $\phi: \mathbb{R}^{p} \times \mathbb{R}^{m} \rightarrow \mathbb{R}^{n}$ and $G: \mathbb{R}_{\geq 0} \times \mathbb{R}^{p} \times \mathbb{R}^{m} \rightarrow \mathbb{R}^{n \times q}$ are also known and they ensure uniqueness and existence of solutions for system (1) for all admissible disturbances.

The aim of this paper is to provide estimations of the state and parameter vectors, i.e. $x$ and $\theta$, respectively; only using the information of the output $y$ and attenuating as much as possible the effects of the external disturbances $w$.

The following assumptions are introduced for the system (1)-(2).

\section{Assumption 1}

The trajectories of the system, the control input, and the external disturbances belong to $\mathcal{L}_{\infty}$, i.e. $\|x\|_{\infty}<+\infty,\|u\|_{\infty}<+\infty$, and $\|w\|_{\infty}<+\infty$, respectively; and $\|G(t, y(t), u(t))\|_{\infty}<+\infty$ for all $t \geq 0$.

Despite the fact that boundedness is assumed, the disturbance $w$ may have a large magnitude; thus, special attenuation techniques have to be applied to provide an acceptable state estimation for system (1). Regarding boundedness of the state $x$, an exact estimate on the state maximum amplitude is not necessary, and since estimation of all variables is achieved in a finite time, the boundedness is also compulsory on a finite interval only. 


\subsection{Preliminaries}

Consider the following nonlinear system

$$
\dot{x}=f(x, w),
$$

where $x \in \mathbb{R}^{n}$ is the state, $w \in \mathbb{R}^{l}$ is the external disturbances, and $f: \mathbb{R}^{n} \times \mathbb{R}^{l} \rightarrow \mathbb{R}^{n}$ is a locally Lipschitz function. For an initial condition $x_{0} \in \mathbb{R}^{n}$ and an external disturbance $w \in \mathcal{L}_{\infty}$, denote the solution by $x\left(t, x_{0}, w\right)$ for any $t \geq 0$ for which the solution exists.

The following stability properties for system (3) are introduced (for more details see [25], [26] and [27]).

\section{Definition 1}

The system (3) is said to be Input-to-State practically Stable (ISpS) if for any $w \in \mathcal{L}_{\infty}$ and any $x_{0} \in \mathbb{R}^{n}$ there exist some functions $\beta \in \mathcal{K} \mathcal{L}, \gamma \in \mathcal{K}$ and a constant $\kappa \in \mathbb{R}_{\geq 0}$ such that

$$
\left\|x\left(t, x_{0}, w\right)\right\| \leq \beta\left(\left\|x_{0}\right\|, t\right)+\gamma\left(\|w\|_{\infty}\right)+\kappa, \forall t \geq 0 .
$$

The system (3) is said to be Input-to-State Stable (ISS) if $\kappa=0$.

These properties also have a Lyapunov function characterization.

\section{Definition 2}

A smooth function $V: \mathbb{R}^{n} \rightarrow \mathbb{R}_{\geq 0}$ is said to be an ISpS Lyapunov function for system (3) if for all $x \in \mathbb{R}^{n}$ and any $w \in \mathcal{L}_{\infty}$ there exist some functions $\psi_{1}, \psi_{2}, \psi_{3} \in \mathcal{K}_{\infty}, \chi \in \mathcal{K}$, and a constant $\kappa \in \mathbb{R}_{\geq 0}$ such that

$$
\begin{gathered}
\psi_{1}(\|x\|) \leq V \leq \psi_{2}(\|x\|), \\
\|x\| \geq \chi\left(\|w\|_{\infty}\right)+\kappa \Rightarrow \nabla V(x) f(x, w) \leq-\psi_{3}(\|x\|) .
\end{gathered}
$$

The function $V$ is said to be an ISS Lyapunov function for system (3) if $\kappa=0$.

\section{Theorem 1}

[26] The system (3) is ISpS (ISS) if and only if it admits an ISpS (ISS) Lyapunov function.

Let us consider the following interconnected nonlinear system

$$
\begin{aligned}
& \dot{x}_{1}=f_{1}\left(x_{1}, x_{2}, w\right), \\
& \dot{x}_{2}=f_{2}\left(x_{1}, x_{2}, w\right),
\end{aligned}
$$

where $x_{i} \in \mathbb{R}^{n_{i}}, w \in \mathbb{R}^{l}$, and $f_{i}: \mathbb{R}^{n_{1}} \times \mathbb{R}^{n_{2}} \times \mathbb{R}^{l} \rightarrow \mathbb{R}^{n_{i}}$ ensures existence of the system solutions at least locally, for $i=1,2$. Suppose that there exist ISpS Lyapunov functions $V_{1}$ and $V_{2}$, for (4) and (5), respectively; such that, for all $x_{i} \in \mathbb{R}^{n_{i}}$ and any $w \in \mathcal{L}_{\infty}$ there exist some functions $\psi_{i 1}, \psi_{i 2}, \psi_{i 3} \in \mathcal{K}_{\infty}, \gamma_{i}, \chi_{i} \in \mathcal{K}$ and some constants $\kappa_{i} \in \mathbb{R}_{\geq 0}$ with $i=1,2$, the following hold

$$
\begin{gathered}
\psi_{i 1}\left(\left\|x_{i}\right\|\right) \leq V_{i}\left(x_{i}\right) \leq \psi_{i 2}\left(\left\|x_{i}\right\|\right), i=1,2, \\
V_{1}\left(x_{1}\right) \geq \max \left[\chi_{1}\left(V_{2}\left(x_{2}\right)\right), \gamma_{1}(\|w\|)+\kappa_{1}\right] \Rightarrow \nabla V_{1}\left(x_{1}\right) f_{1}\left(x_{1}, x_{2}, w\right) \leq-\psi_{13}\left(V_{1}\left(x_{1}\right)\right), \\
V_{2}\left(x_{2}\right) \geq \max \left[\chi_{2}\left(V_{1}\left(x_{1}\right)\right), \gamma_{2}(\|w\|)+\kappa_{2}\right] \Rightarrow \nabla V_{2}\left(x_{2}\right) f_{2}\left(x_{1}, x_{2}, w\right) \leq-\psi_{23}\left(V_{2}\left(x_{2}\right)\right) .
\end{gathered}
$$

Then, the following nonlinear small-gain result is introduced for the interconnected system (4)-(5) in terms of ISpS Lyapunov functions.

\section{Theorem 2}

[25] Suppose that the interconnected system (4)-(5) has ISpS Lyapunov functions $V_{1}$ and $V_{2}$ satisfying the condition (6)-(8). If there exists some constant $\kappa_{0} \in \mathbb{R}_{\geq 0}$ such that

$$
\chi_{1} \circ \chi_{2}(\mathrm{r})<\mathrm{r}, \forall \mathrm{r}>\kappa_{0},
$$

then the interconnected system (4)-(5) is ISpS. The system (4)-(5) is ISS if $\kappa_{0}=\kappa_{1}=\kappa_{2}=0$. 


\section{ADAPTIVE SLIDING-MODE OBSERVER}

Let us introduce the following adaptive observer

$$
\begin{aligned}
\dot{\Omega} & =(A-L C) \Omega+G(t, y, u), \\
\dot{\hat{\theta}} & =\Gamma \Omega^{T} C^{T}\lceil y-C \hat{x}\rfloor^{\alpha}, \\
\dot{\hat{x}} & =A \hat{x}+\phi(y, u)+G(t, y, u) \hat{\theta}+L(y-C \hat{x})+k D \operatorname{sign}[F(y-C \hat{x})]+\Omega \dot{\hat{\theta}},
\end{aligned}
$$

where $\Omega \in \mathbb{R}^{n \times q}$ represents an auxiliary variable, $\hat{\theta} \in \mathbb{R}^{q}$ is the estimation of $\theta$ while $\hat{x} \in \mathbb{R}^{n}$ is the estimation of $x$. The function $\left\lceil\left.\cdot\right|^{\alpha}:=|\cdot|^{\alpha} \operatorname{sign}(\cdot)\right.$, with $|\cdot|$ and $\operatorname{sign}(\cdot)$ understood in the component-wise sense, the function $\operatorname{sign}[q]:=q /\|q\|$ for any vector $q \in \mathbb{R}^{m}$, the observer matrix gain $L \in \mathbb{R}^{n \times p}$ has to be selected such that $(A-L C)$ is Hurwitz and $0<\Gamma^{T}=\Gamma \in \mathbb{R}^{q \times q}$, while $F \in \mathbb{R}^{l \times p}, k, \alpha, \in \mathbb{R}_{\geq 0}$ are designed later. Note that if the signal $G(y, u)$ is persistently exciting (PE), then due to the filtering property of the variable $\Omega$, the variable $C \Omega$ is also PE.

The adaptive sliding-mode observer (10)-(12) represents a modified version of the one proposed in [24]. Such a modification lies in the nonlinear parameter identification algorithm (11) (in [24] just the case when $\alpha=1$ was studied, i.e. the linear case). In this paper, it will be shown that the nonlinear algorithm (11) may improve the rate of convergence and the accuracy of the given estimation. However, from another side, the nonlinearity in (11) also complicates the proof drastically with respect to [24].

In the following, some properties of the nonlinear parameter estimation algorithm (11) are presented but before let us introduce the following assumption.

\section{Assumption 2}

Let $0<\varrho_{\min } \leq \sigma_{\min }(C \Omega(t))$ for all $t \geq 0$ and $\|C \Omega\|_{\infty} \leq \varrho_{\max }<+\infty$.

The existence of $\varrho_{\max }$ follows from Assumption 1 for the Hurwitz matrix $A-L C$ and it cannot be computed a priori in a generic case. The existence of $\varrho_{\min }$ can be assured if the matrix $C \Omega$ has a full column rank, and it is also hard to estimate the value of $\varrho_{\min }$ in advance. However, the values of $\varrho_{\min }$ and $\varrho_{\max }$ can be evaluated numerically during experiments.

\subsection{Nonlinear Parameter Estimation Algorithm}

Let us define the errors $\tilde{\theta}:=\hat{\theta}-\theta$ and $\delta:=x-\hat{x}+\Omega \tilde{\theta}$. Hence, taking into account (10)-(12), the error dynamics are given by

$$
\begin{aligned}
& \dot{\tilde{\theta}}=-\Gamma \Omega^{T} C^{T}\lceil C \Omega \tilde{\theta}-C \delta\rfloor^{\alpha}, \\
& \dot{\delta}=(A-L C) \delta+D(w-k \operatorname{sign}[F(y-C \hat{x})]) .
\end{aligned}
$$

The following lemma shows that the system (13) is ISS with respect to the input $\delta$ for any $\alpha \in[0,1)$.

\section{Lemma 3}

Let Assumption 2 be satisfied. Then, the system (13), with $\alpha \in[0,1)$ and $\Gamma=\Gamma^{T}>0$, is ISS with respect to the input $\delta$. Moreover, its trajectories satisfy the following bounds:

$$
\begin{aligned}
& \|\tilde{\theta}(t)\| \leq \sqrt{2 \lambda_{\max }(\Gamma)}\left(\left(2 \lambda_{\min }(\Gamma)\right)^{\frac{\alpha-1}{2}}\|\tilde{\theta}(0)\|^{1-\alpha}-\frac{2^{\alpha-1} \alpha \varrho_{\min }^{\alpha+1}\left(2 \lambda_{\min }(\Gamma)\right)^{\frac{\alpha+1}{2}}}{2(1+\alpha)} t\right)^{\frac{1}{1-\alpha}}, \forall t \leq T_{\tilde{\theta}}(\tilde{\theta}(0)), \\
& \|\tilde{\theta}(t)\| \leq \sqrt{\frac{\lambda_{\max }(\Gamma)}{\lambda_{\min }(\Gamma)}} \mu_{\tilde{\theta}}, \forall t>T_{\tilde{\theta}}(\tilde{\theta}(0)),
\end{aligned}
$$


with

$$
\begin{gathered}
\mu_{\tilde{\theta}}:=\frac{p^{\frac{1}{\alpha+1}}\left[(1+\alpha)^{2}+2^{2-\alpha} \alpha^{\alpha-1}\right]^{\frac{1}{\alpha+1}}}{\varrho_{\min }(1+\alpha)^{\frac{\alpha}{\alpha+1}}}\|C\|\|\delta\|_{\infty} \\
T_{\tilde{\theta}}(\tilde{\theta}(0)) \leq \max \left[0, \frac{2(1+\alpha)\left(\left(2 \lambda_{\min }(\Gamma)\right)^{\frac{\alpha-1}{2}}\|\tilde{\theta}(0)\|^{1-\alpha}-\left(2 \lambda_{\max }(\Gamma)\right)^{\frac{\alpha-1}{2}} \mu_{\tilde{\theta}}^{1-\alpha}\right)}{2^{\alpha-1} \alpha \varrho_{\min }^{\alpha+1}\left(2 \lambda_{\min }(\Gamma)\right)^{\frac{\alpha+1}{2}}}\right],
\end{gathered}
$$

and any $\tilde{\theta}(0) \in \mathbb{R}^{q}$.

The proofs of all results are postponed to the Appendix.

Hence, it is concluded that the solutions of system (13) are ultimately bounded with its trajectories satisfying the bounds given by (15) and (16) for any $\alpha \in[0,1)$. Moreover, some important ISS properties with respect to the input $\delta$ are provided for the system (13).

\section{Remark 1}

Lemma 3 shows that the solutions of the system (13) enter into the bound (16) at most in a finite time $T_{\tilde{\theta}}(\tilde{\theta}(0))$ for any $\alpha \in[0,1)$. In addition, the parameter estimation error may be reduced according to the choice of the parameter $\alpha \in[0,1)$ since the size of $\mu_{\tilde{\theta}}$ depends on this value.

Now, the following lemma shows that system (14) is ISS with respect to the inputs $\tilde{\theta}$ and $w$.

\section{Lemma 4}

Let Assumption 1 be satisfied. Suppose that the following matrix inequalities

$$
\begin{gathered}
(A-L C)^{T} P+P(A-L C)+\beta^{-1} P+(\beta r+2 \varpi) C^{T} C \leq 0, \\
P D-C^{T} F^{T} \leq 0,
\end{gathered}
$$

are feasible for a matrix $0<P^{T}=P \in \mathbb{R}^{n \times n}$, matrices $F \in \mathbb{R}^{l \times p}, L \in \mathbb{R}^{n \times p}$, and constants $\beta, r, \varpi>0$, then the system (14), with $k=\|w\|_{\infty}$, is ISS with respect to the inputs $\tilde{\theta}$ and $w$. Moreover, its trajectories satisfy the following bounds:

$$
\begin{aligned}
& \|\delta(t)\| \leq e^{-\frac{\xi_{1}}{2} t} \sqrt{\frac{\lambda_{\max }(P)}{\lambda_{\min }(P)}}\|\delta(0)\|, \forall t \leq T_{\delta}(\delta(0)), \\
& \|\delta(t)\| \leq \sqrt{\frac{\lambda_{\max }(P)}{\lambda_{\min }(P)}} \mu_{\delta}, \forall t>T_{\delta}(\tilde{\theta}(0)),
\end{aligned}
$$

with

$$
\begin{gathered}
\zeta_{1}:=\frac{(1-\rho)\left(\beta^{-1} \lambda_{\min }(P)+\varpi\|C\|^{2}\right)}{\lambda_{\max }(P)}, \\
\mu_{\delta}:=\frac{\sqrt{\varpi} \varrho_{\max }}{\sqrt{\rho\left(\beta^{-1} \lambda_{\min }(P)+\varpi\|C\|^{2}\right)}}\|\tilde{\theta}\|_{\infty}+\mu_{\delta w}, \quad \mu_{\delta w}:=\frac{2\|F\|}{\sqrt{\rho\left(\beta^{-1} \lambda_{\min }(P)+\varpi\|C\|^{2}\right)}}\|w\|_{\infty}, \\
T_{\delta}(\delta(0)) \leq \max \left(0, \frac{2\left[\ln (\|\delta(0)\|)-\ln \left(\sqrt{\frac{\lambda_{\min }(P)}{\lambda_{\max }(P)}} \mu_{\delta}\right)\right]}{\zeta_{1}}\right),
\end{gathered}
$$

$\rho \in(0,1)$, and any $\delta(0) \in \mathbb{R}^{n}$.

Note that in order to attenuate the effects of the external disturbances $w$, the size of the parameter $\mu_{\delta w}$ could be minimized. 


\subsection{Convergence of the Adaptive Observer}

In the following, based on the statements given by Lemmas 3, 4, and small-gain arguments, it will be shown that the interconnected error system (13)-(14) is ISS with respect to the external disturbance $w$, for any $\alpha \in[0,1)$.

\section{Theorem 5}

Let Assumptions 1 and 2 be satisfied. Suppose that the matrix inequalities (17)-(18) and

$$
\left(\frac{p^{\frac{2}{\alpha+1}}\left[(1+\alpha)^{2}+2^{2-\alpha} \alpha^{\alpha-1}\right]^{\frac{2}{\alpha+1}}\|C\|^{2} \sqrt{\beta \varpi} \varrho_{\max } \lambda_{\min }(\Gamma)}{\varrho_{\min }^{2}(1+\alpha)^{\frac{2 \alpha}{\alpha+1}} \lambda_{\max }(P) \lambda_{\max }(\Gamma) \sqrt{\rho \lambda_{\min }(P)}}\right)<1,
$$

are feasible for matrices $0<P^{T}=P \in \mathbb{R}^{n \times n}, \quad 0<\Gamma^{T}=\Gamma \in \mathbb{R}^{q \times q}, \quad F \in \mathbb{R}^{l \times p}, \quad L \in \mathbb{R}^{n \times p}$, constants $\beta, r, \varpi, p>0, \alpha \in[0,1)$, and $\rho \in(0,1)$, then the interconnected error system (13)-(14) is ISS with respect to the input $w$. Moreover, with no external disturbances, i.e. $w=0$, the system (13)-(14) is globally asymptotically stable.

Note that Theorem 5 implies that the estimation error $e:=x-\hat{x}=\delta+\Omega \tilde{\theta}$ is also ISS since

$$
\|e(t)\| \leq(1+\|\Omega\|)\left\|\left(\begin{array}{c}
\tilde{\theta}(t) \\
\delta(t)
\end{array}\right)\right\|, \forall t \geq 0,
$$

for any $\alpha \in[0,1)$.

After the statements given by Lemmas 3 and 4; and Theorem 5, one can highlight the following points:

1. For the ideal case, i.e. $w=0$ with $k=0$, the estimations $\hat{\theta}$ and $\hat{x}$ converge to the real values $\theta$ and $x$, respectively; and the rate of convergence for $\tilde{\theta}$ is faster than exponential, i.e. faster than the classic linear algorithm, for any $\alpha \in[0,1)$, which is our motivation for designing of such a nonlinear estimation scheme.

2. For the perturbed case, i.e. $w \neq 0$, one can show by taking $V_{e}=e^{T} P e$ that

$$
\begin{array}{r}
\dot{V}_{e} \leq e^{T}\left[(A-L C)^{T} P+P(A-L C)\right] e+2 e^{T} P\left(\Omega \Gamma \Omega^{T} C^{T}\lceil C e]^{\alpha}+G(t, y, y) \tilde{\theta}\right) \\
+2 e^{T} C^{T} F^{T}(w-k \operatorname{sign}[F C e]),
\end{array}
$$

and therefore, if one fixes $k=\|w\|_{\infty}$, the effect of the external disturbance $w$ is completely attenuated.

3. The condition (18) introduces structural restrictions over the triple $(A, D, C)$; specifically, it must not have invariant zeros, and the relative degree of the output $y$ with respect to the input $w$ must be equal to one. In order to avoid these restrictions some approaches are proposed in [28] and [29].

4. To find a solution of the matrix inequality (17), one can rewrite it as follows

$$
(A-L C)^{T} P+P(A-L C)+\tau_{1} P+\tau_{2} C^{T} C \leq 0,
$$

where $\tau_{1}=\beta^{-1}$ and $\tau_{2}=\beta r+2 \varpi$ are new variables. Then, using the fact that $X Y^{T}+$ $Y X^{T} \leq X \Lambda^{-1} X^{T}+Y \Lambda Y^{T}$ holds for every $X \in \mathbb{R}^{k \times n}, Y \in \mathbb{R}^{k \times n}$, and $0<\Lambda=\Lambda^{T} \in$ $\mathbb{R}^{n \times n}$ (see, for instance [30]); and applying Schur's complement, it follows that

$$
\left(\begin{array}{ccc}
A^{T} P+P A-C^{T} Y^{T}-Y C+\tau_{2} C^{T} C & \frac{\tau_{1}}{2} I_{n} & P \\
\frac{\tau_{1}}{2} I_{n} & -\Lambda^{-1} & 0 \\
P & 0 & -\Lambda
\end{array}\right) \leq 0
$$

is equivalent to (23) for any fixed $0<\Lambda^{T}=\Lambda \in \mathbb{R}^{n \times n}$ and $Y=P L \in \mathbb{R}^{n \times p}$. Note that (24) is now a linear matrix inequality with respect to matrices $P$ and $Y$, and parameters $\tau_{1}$ and $\tau_{2}$; respectively. One can compute the design matrix $L=P^{-1} Y$. Then, the matrix inequality (21) can be numerically verified with the corresponding values of the solution of (24) and fixing $\alpha$ and $\rho$. 
5. The feasibility of (24) is ensured for sufficiently small $\tau_{1}$ and $\tau_{2}$, due to the fact that the pair $(A, C)$ is observable.

6. The parameter $\mu_{\delta w}$ can be written, in terms of $\tau_{1}$ and $\tau_{2}$, as follows

$$
\mu_{\delta w}=\frac{\sqrt{8}\|F\|}{\sqrt{\rho}\left(2 \tau_{1} \lambda_{\min }(P)+\tau_{2}\|C\|^{2}-\frac{r}{\tau_{1}}\|C\|^{2}\right)^{\frac{1}{2}}},
$$

with the constraint $r<\tau_{1} \tau_{2}$ to ensure that $\varpi>0$. Then, in order to minimize the size of $\mu_{\delta w}$, i.e. attenuate the effects of the external disturbances $w$, it is possible to propose de following optimization problem.

Optimization Problem. The minimization of $\mu_{\delta w}$, for a fixed $\rho \in(0,1)$ and a matrix $F$ satisfying (18), is equivalent to maximize $\lambda_{\min }(P), \tau_{1}$ and $\tau_{2}$, i.e.

$$
\gamma, \tau_{1}, \tau_{2} \rightarrow \max _{P, F, Y, \gamma, \tau_{1}, \tau_{2}}
$$

subject to (18), (24) and $\gamma I \leq P$.

\section{SIMULATION RESULTS}

\subsection{Duffing's Oscillator}

Let us consider the following exited Duffing system

$$
\begin{aligned}
& \dot{x}=\left(\begin{array}{cc}
0 & 1 \\
1 & -\mu
\end{array}\right) x+\left(\begin{array}{l}
0 \\
u
\end{array}\right)+\left(\begin{array}{c}
0 \\
-y^{3}
\end{array}\right) \theta+\left(\begin{array}{l}
1 \\
0
\end{array}\right) w, \\
& y=x_{1}
\end{aligned}
$$

where $u=0.3 \cos (t), w=0.5 \sin (2 t), \mu=0.2, \theta=3$ and $x(0)=(2,1)^{T}$. For these parameters, the system develops a chaotic behavior and Assumption 1 is clearly satisfied. Let us apply the statements given by Theorem 5 for both cases, i.e. the ideal and the perturbed case.

Let us fix the matrix $\Gamma=100 I_{2}$ and the gain $k=1$. Then, SeDuMi solver among YALMIP in Matlab is used to find a solution for the LMIs (18), (24), and (21), respectively. The following feasible solution, with $\Lambda=I_{2}$, is found

$$
\begin{gathered}
P=\left(\begin{array}{cc}
28.6444 & 0 \\
0 & 0.4455
\end{array}\right), L=\left(\begin{array}{l}
30.5749 \\
64.3579
\end{array}\right), F=28.6444, \\
\tau_{1}=0.0281, \tau_{2}=604.7644, \beta=35.6235, r=15.2789, \varpi=30.2382 .
\end{gathered}
$$

For this matrix $L$ and the given $G(t, y, u)$, it is possible to show, after some simulations, that Assumption 2 is satisfied. All the simulations have been done in Matlab with the Euler discretization method, sample time equal to 0.001 , and initial conditions $\Omega(0)=(0,0)^{T}, \hat{\theta}(0)=0$ and $\hat{x}(0)=(0,0)^{T}$. The results for the ideal case, i.e. $w=0$, with $\alpha=0.0,0.5$ (nonlinear algorithm) and $\alpha=1.0$ (linear algorithm) for the parameter estimation algorithm, are depicted by Figure 1. One can see that the estimations $\hat{\theta}$ and $\hat{x}$, given by the nonlinear algorithm, converge to the real values $\theta$ and $x$, respectively; faster than the linear algorithm.

The results for the perturbed case, i.e. $w=0.5 \sin (2 t)$, with $\alpha=0.0,0.5$ (nonlinear algorithm) and $\alpha=1.0$ (linear algorithm) for the parameter estimation algorithm, are depicted by Figure 2. In this case, the estimations $\hat{\theta}$ and $\hat{x}$ converge to a neighborhood of the real values $\theta$ and $x$, respectively. One may see that the nonlinear algorithm still converges, to a neighborhood of the real value, faster than the linear algorithm.

Now, some results with different values of $\alpha$ are presented in order to show how the estimation error $\left(\tilde{\theta}, e^{T}\right)^{T}$ is improved with respect to the choice of $\alpha$. The results, for the ideal and perturbed 

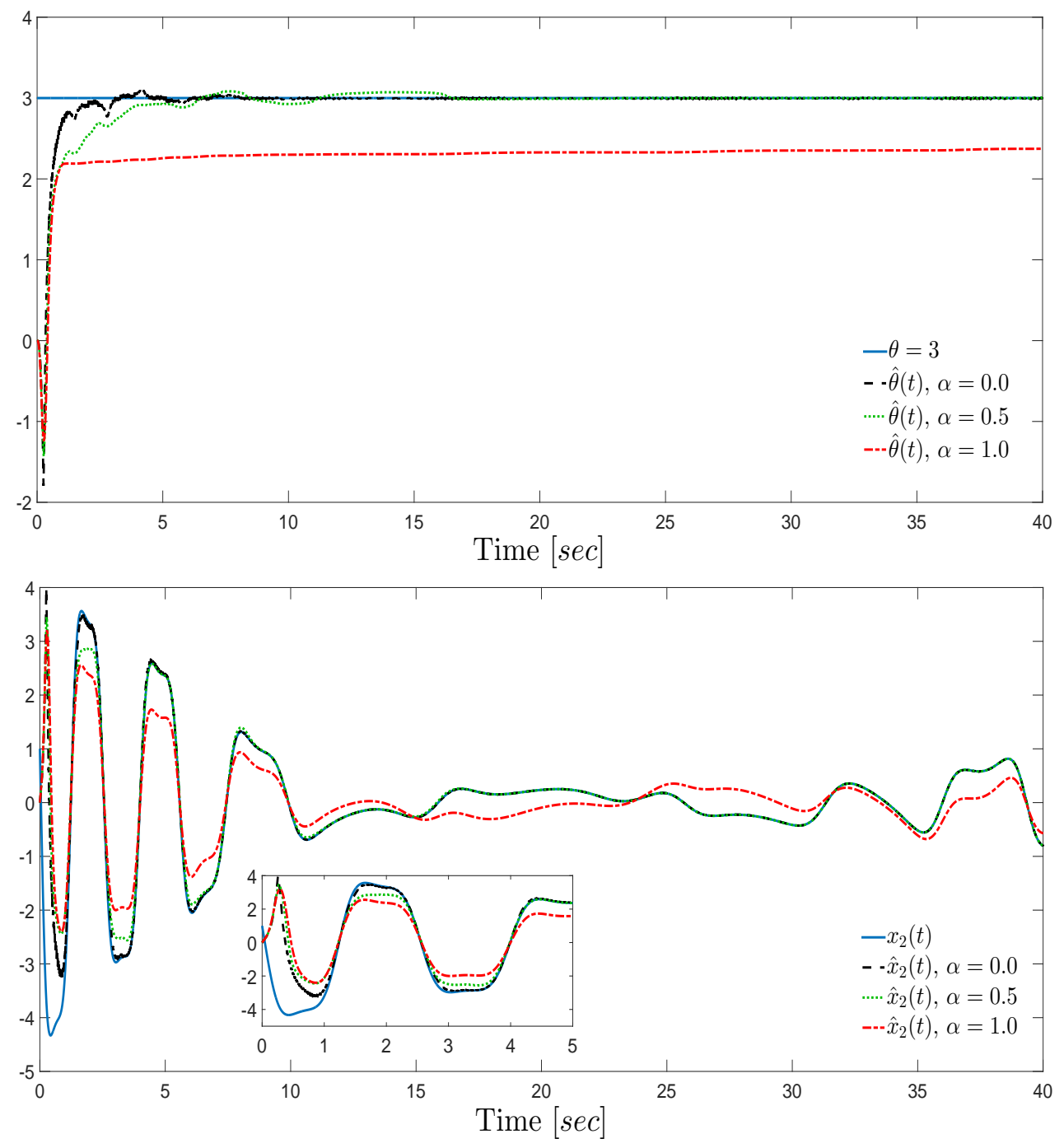

Figure 1. The estimations of $\hat{\theta}$ and $\hat{x}$ - Ideal Case $(\alpha=0.0, \alpha=0.5$ and $\alpha=1.0)$

case, are depicted by Figure 3. It is possible to see that there exists a value of $\alpha$, in this case $\alpha=0.5$, such that the size of the estimation error bound (22) is minimized. One can also see that the nonlinear algorithm, i.e. $\alpha \in[0,1)$, may increase the precision of the estimation with respect to the linear algorithm, i.e. $\alpha=1$.

\subsection{Chua's Oscillator}

Consider the following Chua system

$$
\begin{aligned}
& \dot{x}=\left(\begin{array}{ccc}
0 & 0 & 0 \\
1 & -1 & 1 \\
0 & 0 & 0
\end{array}\right) x+\left(\begin{array}{cc}
c y_{1}+y_{2}-y_{1}^{3} & 0 \\
0 & 0 \\
0 & -y_{2}
\end{array}\right) \theta+\left(\begin{array}{l}
0 \\
1 \\
0
\end{array}\right) w, \\
& y=\left(\begin{array}{ll}
x_{1} & x_{2}
\end{array}\right)^{T},
\end{aligned}
$$

where $w=0.5(\sin (2 t)+2 \sin (0.2 t)+0.1), c=0.5, \theta=(16,10)^{T}$ and $x(0)=(3.9,-3.2,0.03)^{T}$. For these parameters, the system develops a chaotic behavior and Assumption 1 is also satisfied. Let us apply again the statements given by Theorem 5 for both cases, i.e. the ideal and the perturbed case. 

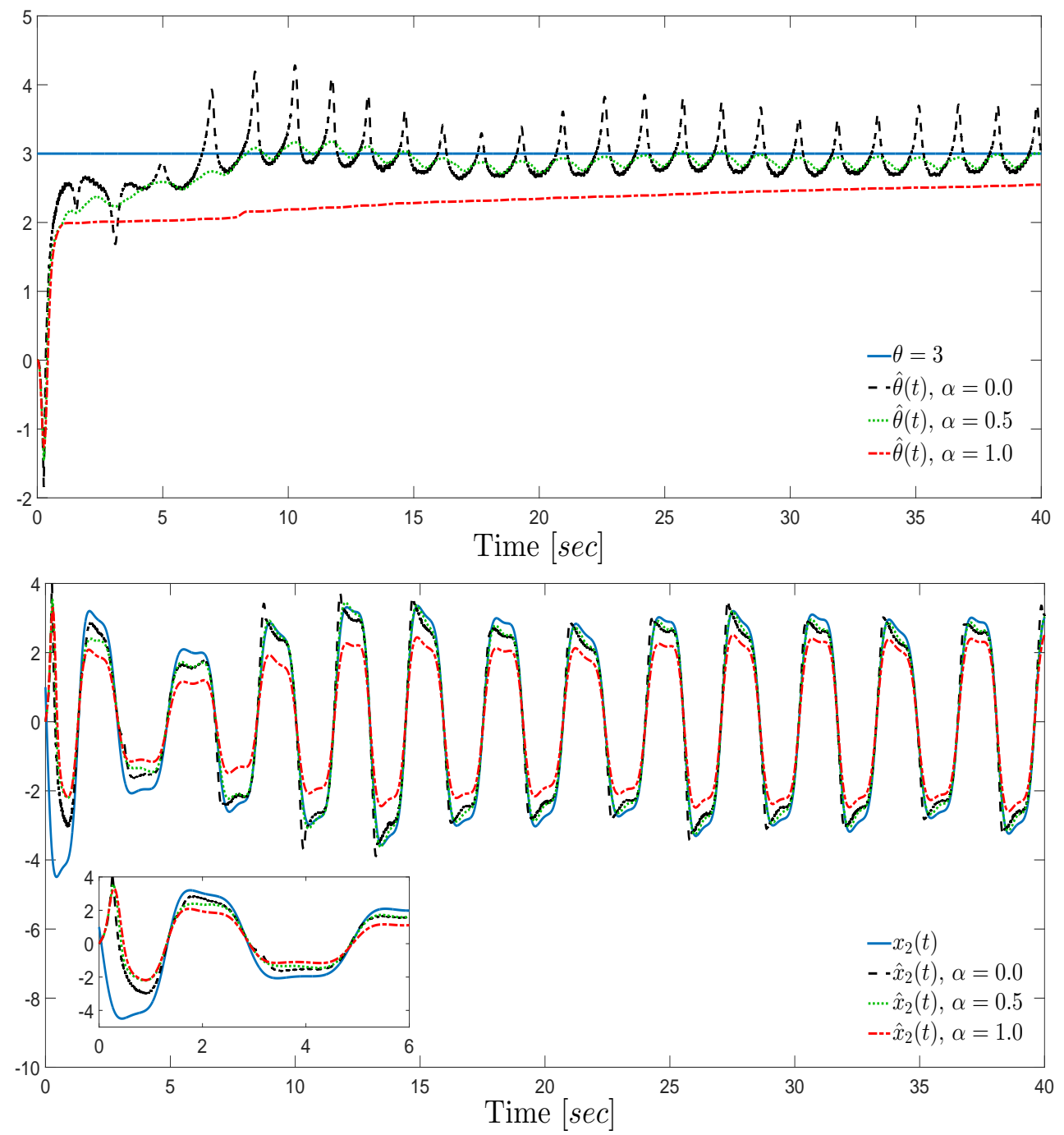

Figure 2. The estimations of $\hat{\theta}$ and $\hat{x}$ - Perturbed Case $(\alpha=0.0, \alpha=0.5$ and $\alpha=1)$

Fixing the matrix $\Gamma=100 I_{3}$ and the gain $k=1$. Then, SeDuMi solver among YALMIP in Matlab is used to find a solution for the LMIs (18), (24), and (21), respectively. The following feasible solution, with $\Lambda=I_{3}$, is found

$$
\begin{gathered}
P=\left(\begin{array}{ccc}
15.3245 & 0.3386 & 0.0107 \\
0.3386 & 18.4631 & 0.0000 \\
0.0107 & 0.0000 & 0.0706
\end{array}\right), L=\left(\begin{array}{cc}
2.1356 & -0.0640 \\
0.1268 & 2.0852 \\
0.1307 & 25.6953
\end{array}\right), F=\left(\begin{array}{ll}
0.3386 & 18.4631
\end{array}\right), \\
\tau_{1}=0.1478, \tau_{2}=294.9623, \beta=6.7652, r=39.2397, \varpi=14.7481 .
\end{gathered}
$$

For this matrix $L$ and the given $G(t, y, u)$, one can show, after some simulations, that Assumption 2 is also satisfied. The simulations have been done in Matlab with initial conditions $\Omega(0)=\mathbf{0}_{3 \times 2}$, $\hat{\theta}(0)=(0,0)^{T}$ and $\hat{x}(0)=(0,0,0)^{T}$. The results for the ideal case, i.e. $w=0$, with $\alpha=0.0,0.5$ (nonlinear algorithm) and $\alpha=1.0$ (linear algorithm) for the parameter estimation algorithm, are depicted by Figure 4 . One can see that the estimations $\hat{\theta}$ and $\hat{x}$, given by the nonlinear algorithm, converge to the real values $\theta$ and $x$, respectively; faster than the linear algorithm.

The results for the perturbed case, i.e. $w=0.5(\sin (2 t)+2 \sin (0.2 t)+0.1)$, with $\alpha=0.0,0.5$ (nonlinear algorithm) and $\alpha=1.0$ (linear algorithm) for the parameter estimation algorithm, are 

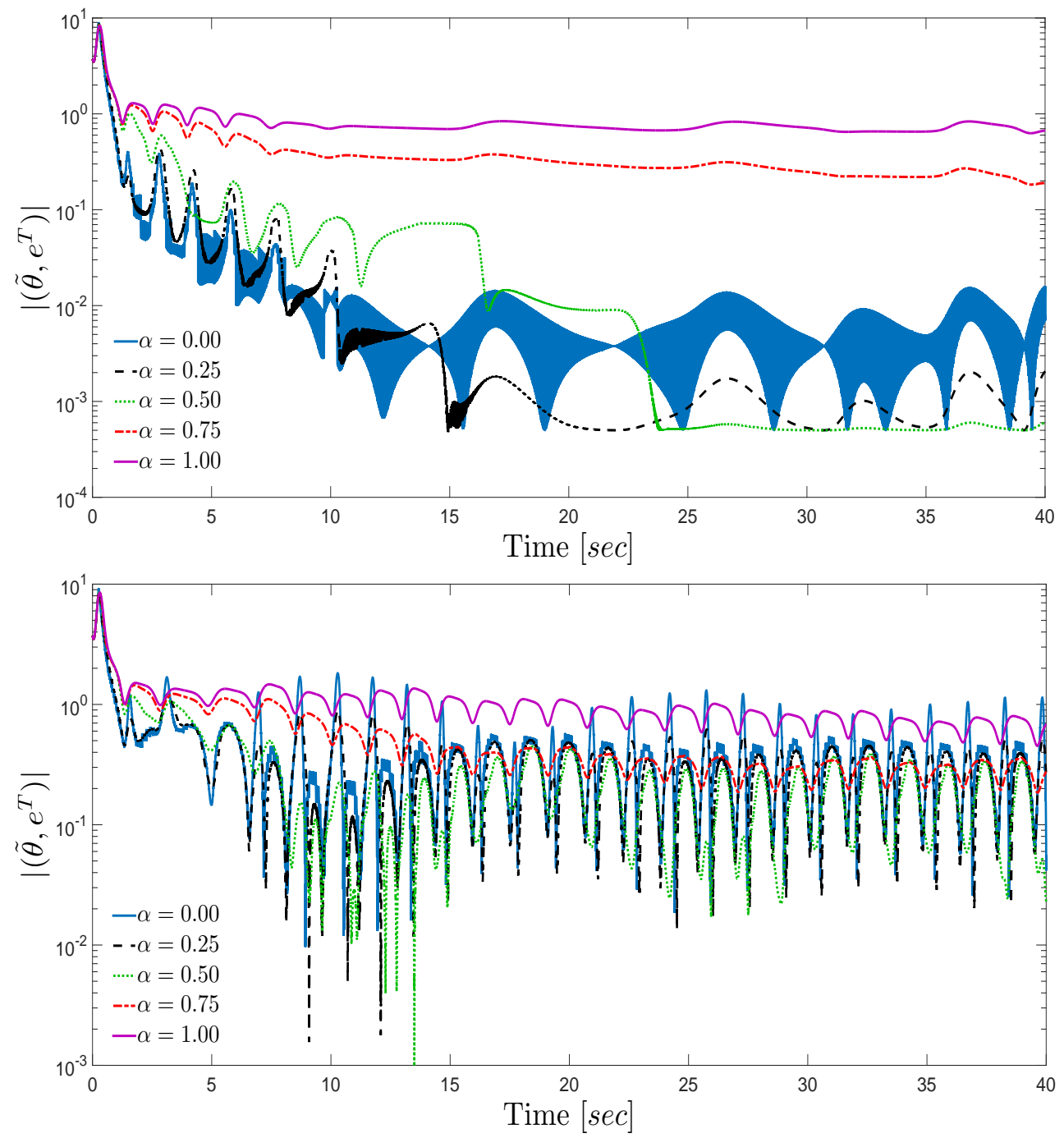

Figure 3. Estimation Error for different values of $\alpha$. The top graph shows the estimation error for the ideal case, i.e. $w=0$; while the bottom graph illustrates the perturbed case, i.e. $w=0.5 \sin (2 t)$.

depicted by Figure 5. In this case, the estimations $\hat{\theta}$ and $\hat{x}$ converge to a neighborhood of the real values $\theta$ and $x$, respectively. One may see that the nonlinear algorithm still converges, to a neighborhood of the real value, faster than the linear algorithm.

Some results with different values of $\alpha$ are also presented in order to show how the estimation error $\left(\tilde{\theta}, e^{T}\right)^{T}$ is improved with respect to the choice of $\alpha$. The results, for the ideal and perturbed case, are depicted by Figure 6. It is possible to see that there exists a value of $\alpha$, in this case $\alpha=0.5$, such that the size of the estimation error bound (22) is minimized. One can also see that the nonlinear algorithm, i.e. $\alpha \in[0,1)$, may increase the precision of the estimation with respect to the linear algorithm, i.e. $\alpha=1$.

\section{CONCLUSIONS}

In this paper an adaptive sliding-mode observer based on a nonlinear parameter estimation algorithm is proposed for uncertain nonlinear systems. The given adaptive sliding-mode observer is a modified version of that one proposed by [24]. Such a modification lies in the inclusion of a nonlinear 

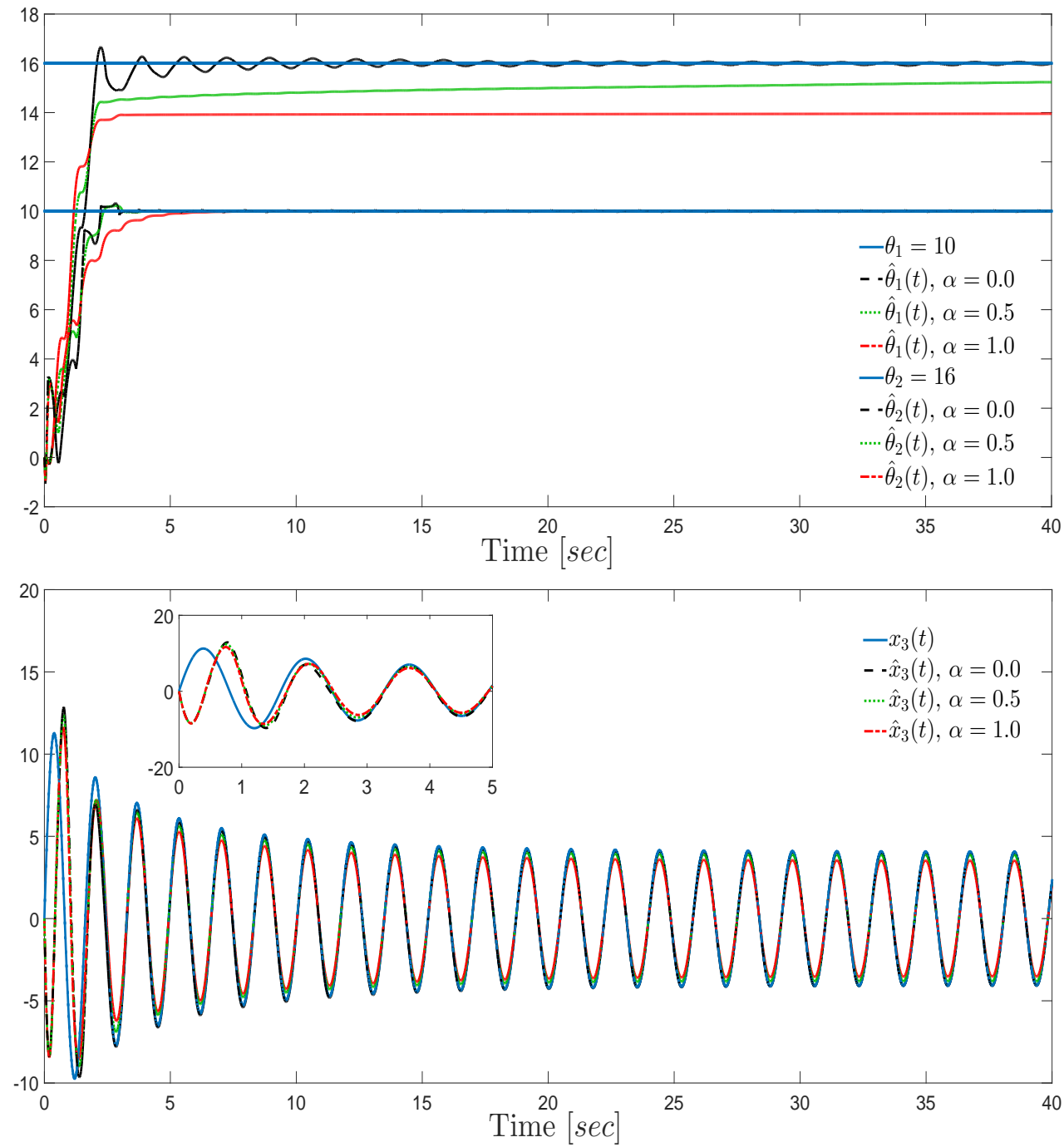

Figure 4. The estimations of $\hat{\theta}$ and $\hat{x}$ - Ideal Case $(\alpha=0.0, \alpha=0.5$ and $\alpha=1.0)$

parameter estimation algorithm that provides a rate of convergence faster than exponential, i.e. faster than the classic linear algorithm. Then, the proposed parameter estimation algorithm is included in the structure of a sliding-mode state observer providing an ultimate bound for the state and parameter estimation error. This solution ensures that the level of observer robustness with respect to some matched disturbances is improved as well as the rate of convergence. The corresponding proofs of convergence are developed based on Lyapunov function approach and input-to-state stability theory. Simulation results illustrate the efficiency of the proposed adaptive sliding-mode observer.

\section{ACKNOWLEDGEMENT}

H. Ríos gratefully acknowledge the financial support from CONACYT 270504. This work was also supported in part by HoTSMoCE Inria associate team program, by ANR Finite4SoS (ANR 15 CE23 0007), by the Government of Russian Federation (Grant 074-U01) and the Ministry of Education and Science of Russian Federation (Project 14.Z50.31.0031). 

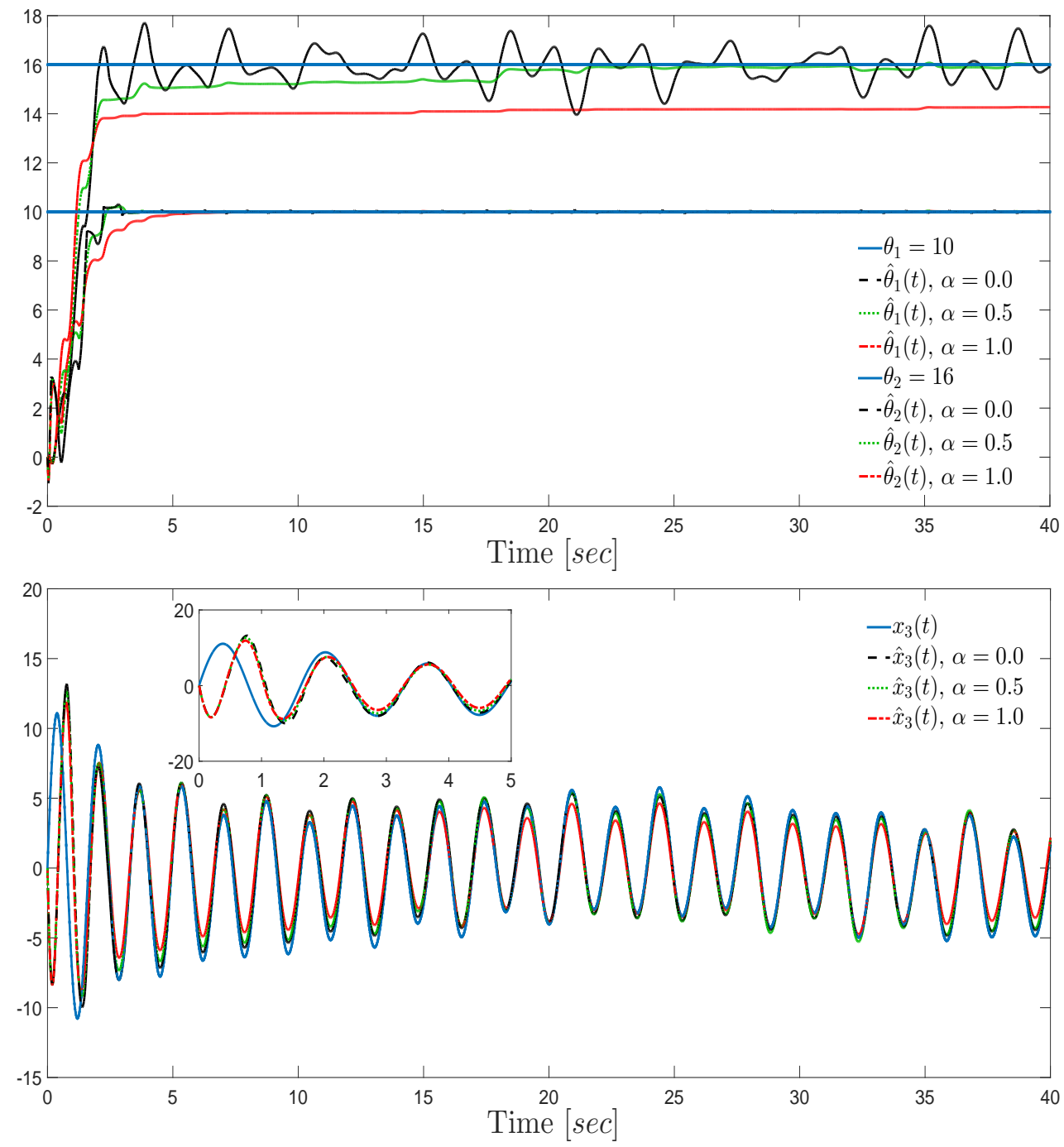

Figure 5. The estimations of $\hat{\theta}$ and $\hat{x}$ - Perturbed Case $(\alpha=0.0, \alpha=0.5$ and $\alpha=1)$

\section{APPENDIX}

Let us consider the nonlinear system (3), i.e.

$$
\dot{x}=f(x, w)
$$

where $x \in \mathbb{R}^{n}$ is the state, $w \in \mathbb{R}^{l}$ is the external disturbances, and $f: \mathbb{R}^{n} \times \mathbb{R}^{l} \rightarrow \mathbb{R}^{n}$ ensures existence of the system solutions at least locally. The following preliminary result describes some ISS properties for system (3) and quadratic Lyapunov functions.

\section{Lemma 6}

Let $V: \mathbb{R}^{n} \rightarrow \mathbb{R}_{\geq 0}$ be a smooth function. If there exist some positive constants $\psi_{1}, \psi_{2}, \psi_{3}, \psi_{4}>0$ and $\gamma \in(0,1]$ such that

$$
\begin{gathered}
\psi_{1}\|x\|^{2} \leq V(x) \leq \psi_{2}\|x\|^{2}, \\
\nabla V(x) f(x, w) \leq-\psi_{3} V^{\gamma}(x), \forall\|x\| \geq \mu:=\psi_{4}\|w\|_{\infty},
\end{gathered}
$$



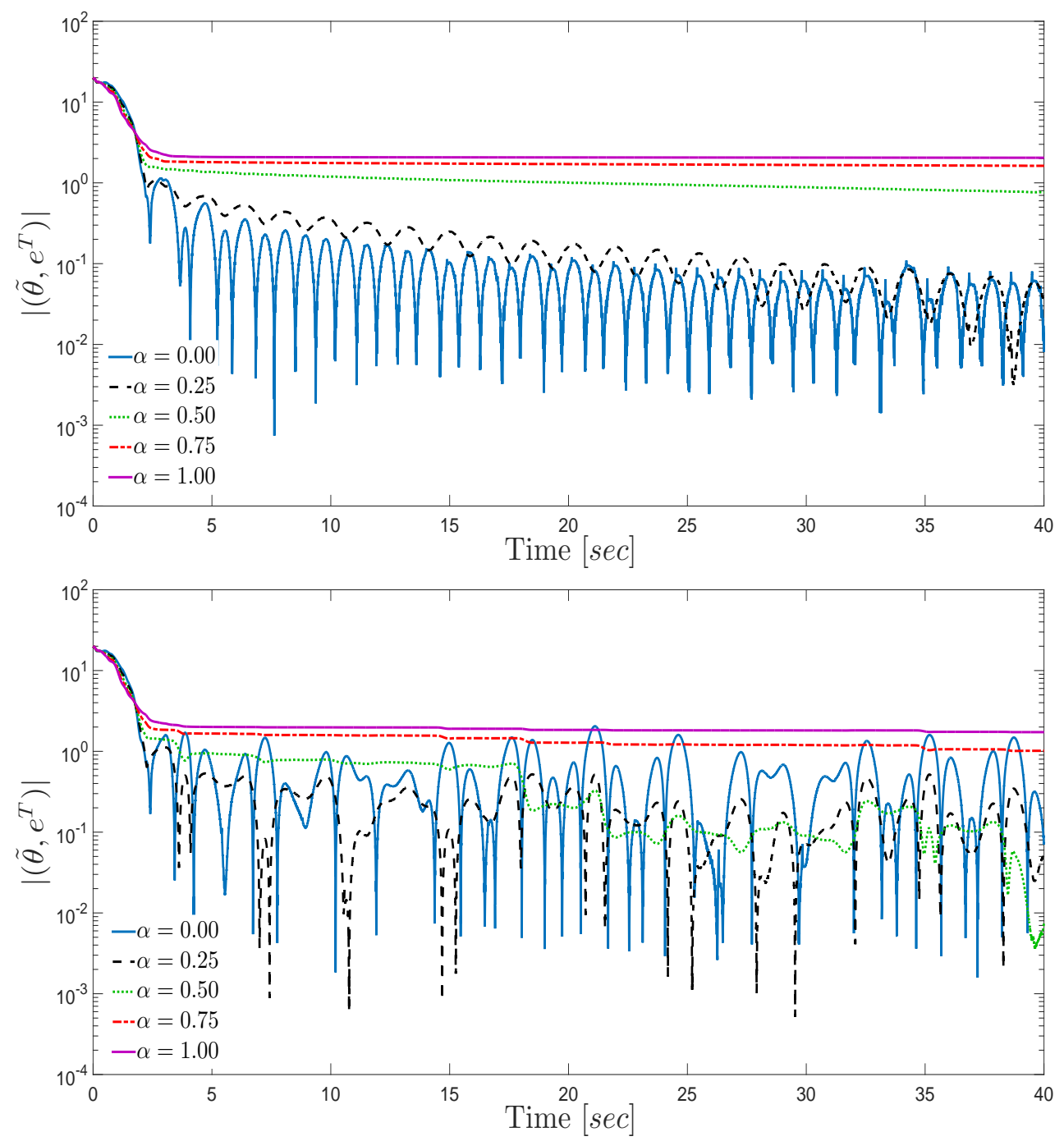

Figure 6. Estimation Error for different values of $\alpha$. The top graph shows the estimation error for the ideal case, i.e. $w=0$; while the bottom graph illustrates the perturbed case, i.e. $w=0.5(\sin (2 t)+2 \sin (0.2 t)+$ $0.1)$.

then the system (3) is ISS with respect to the input $w$. Moreover, its trajectories satisfy the following bounds

$$
\begin{aligned}
& \left\|x\left(t, x_{0}, w\right)\right\| \leq\left\{\begin{array}{ll}
\frac{1}{\sqrt{\psi_{1}}}\left(\psi_{2}^{1-\gamma}\left\|x_{0}\right\|^{2(1-\gamma)}-\psi_{3}(1-\gamma) t\right)^{\frac{1}{2(1-\gamma)}}, & \text { for } \gamma \in(0,1), \\
e^{-\frac{\psi_{3}}{2} t} \sqrt{\frac{\psi_{2}}{\psi_{1}}}\left\|x_{0}\right\|, & \text { for } \gamma=1,
\end{array} \quad\right. \\
& \left\|x\left(t, x_{0}, w\right)\right\| \leq \sqrt{\frac{\psi_{2}}{\psi_{1}}} \mu, \forall t>T\left(x_{0}\right),
\end{aligned}
$$

where

$$
T\left(x_{0}\right) \leq \begin{cases}\max \left(0, \frac{\psi_{2}^{1-\gamma}\left\|x_{0}\right\|^{2(1-\gamma)}-\psi_{1}^{1-\gamma} \mu^{2(1-\gamma)}}{\psi_{3}(1-\gamma)}\right), & \text { for } \gamma \in(0,1), \\ \max \left(0, \frac{2\left[\ln \left(\left\|x_{0}\right\|\right)-\ln \left(\sqrt{\frac{\psi_{1}}{\psi_{2}}} \mu\right)\right]}{\psi_{3}}\right), & \text { for } \gamma=1\end{cases}
$$

Proof

According to Definition 2, the function $V$ is an ISS Lyapunov function. Therefore, from Theorem 1, the 
system (3) is ISS with respect to the input $w$. Then, from (27) and the comparison principle (see, e.g. [31]), it follows that

$$
V(t) \leq \begin{cases}\left(V^{1-\gamma}(0)-\psi_{3}(1-\gamma) t\right)^{\frac{1}{1-\gamma}}, & \text { for } \gamma \in(0,1), \quad \forall t \leq T\left(x_{0}\right) \\ e^{-\psi_{3} t} V(0), & \text { for } \gamma=1\end{cases}
$$

and using (26), one gets the upper bound (28); and consequently (29) is obtained. Note that (28) and (29) hold for any $x_{0} \in \mathbb{R}^{n}$, with no restriction on how large $\mu$ is. The calculation of the time $T\left(x_{0}\right)$ is straightforward from the upper bound (28).

Proof of Lemma 3: Let us consider the candidate Lyapunov function

$$
V_{\tilde{\theta}}=\frac{1}{2} \tilde{\theta}^{T} \Gamma^{-1} \tilde{\theta}
$$

which satisfies the following inequalities

$$
\begin{gathered}
c_{1}^{-1}\|\tilde{\theta}\|^{2} \leq V_{\tilde{\theta}} \leq c_{2}^{-1}\|\tilde{\theta}\|^{2}, \\
c_{1}^{-\frac{\alpha+1}{2}}\|\tilde{\theta}\|^{\alpha+1} \leq V_{\tilde{\theta}}^{\frac{\alpha+1}{2}} \leq c_{2}^{-\frac{\alpha+1}{2}}\|\tilde{\theta}\|^{\alpha+1},
\end{gathered}
$$

where $c_{1}:=2 \lambda_{\max }(\Gamma)$ and $c_{2}:=2 \lambda_{\min }(\Gamma)$. The time derivative of $V_{\tilde{\theta}}$ along the trajectories of (13) is given by

$$
\begin{aligned}
\dot{V}_{\tilde{\theta}} & =\tilde{\theta}^{T} \Gamma^{-1}\left(-\Gamma \Omega^{T} C^{T}\lceil C \Omega \tilde{\theta}-C \delta\rfloor^{\alpha}\right), \\
& =-\tilde{\theta}^{T} \Omega^{T} C^{T}\lceil C \Omega \tilde{\theta}-C \delta\rfloor^{\alpha} .
\end{aligned}
$$

Using Young's inequality it is derived:

$$
\tilde{\theta}^{T} \Omega^{T} C^{T}\lceil C \Omega \tilde{\theta}-C \delta\rfloor^{\alpha} \geq \sum_{i=1}^{p}\left|(C \Omega \tilde{\theta})_{i}\left\|(C \Omega \tilde{\theta})_{i}-\left.(C \delta)_{i}\right|^{\alpha}-\frac{2 \alpha^{\alpha}}{(1+\alpha)^{1+\alpha}}\right\| C \delta \|_{\alpha+1}^{\alpha+1},\right.
$$

and applying Jensen's and Young's inequalities one gets:

$$
\begin{gathered}
\tilde{\theta}^{T} \Omega^{T} C^{T}\lceil C \Omega \tilde{\theta}-C \delta\rfloor^{\alpha} \geq 2^{\alpha-1} \sum_{i=1}^{p}\left|(C \Omega \tilde{\theta})_{i}\right|\left(\left|(C \Omega \tilde{\theta})_{i}\right|^{\alpha}-\left|(C \delta)_{i}\right|^{\alpha}\right)-\frac{2 \alpha^{\alpha}}{(1+\alpha)^{1+\alpha}}\|C \delta\|_{\alpha+1}^{\alpha+1} \\
\geq 2^{\alpha-1} \frac{\alpha}{1+\alpha} \sum_{i=1}^{p}\left|(C \Omega \tilde{\theta})_{i}\right|^{\alpha+1}-\frac{2 \alpha}{1+\alpha}\left(2^{\alpha-2}+\frac{\alpha^{\alpha-1}}{(1+\alpha)^{\alpha}}\right)\|C \delta\|_{\alpha+1}^{\alpha+1} \\
=2^{\alpha-1} \frac{\alpha}{1+\alpha}\|C \Omega \tilde{\theta}\|_{\alpha+1}^{\alpha+1}-\frac{2 \alpha}{1+\alpha}\left(2^{\alpha-2}+\frac{\alpha^{\alpha-1}}{(1+\alpha)^{\alpha}}\right)\|C \delta\|_{\alpha+1}^{\alpha+1} .
\end{gathered}
$$

By relations between vector norms, $\|C \Omega \tilde{\theta}\| \leq\|C \Omega \tilde{\theta}\|_{\alpha+1}$ holds since $2>\alpha+1$, and $\|C \delta\|_{\alpha+1} \leq$ $p^{\frac{1}{\alpha+1}}\|C \delta\|_{\infty}$. Therefore, one finally obtains:

$$
\dot{V}_{\tilde{\theta}} \leq-2^{\alpha-1} \frac{\alpha}{1+\alpha}\|C \Omega \tilde{\theta}\|^{\alpha+1}+\frac{2 p \alpha}{1+\alpha}\left(2^{\alpha-2}+\frac{\alpha^{\alpha-1}}{(1+\alpha)^{\alpha}}\right)\|C \delta\|_{\infty}^{\alpha+1},
$$

and taking into account Assumption 2, one obtains that $\dot{V}_{\tilde{\theta}}$ is upper bounded as follows:

$$
\dot{V}_{\tilde{\theta}} \leq-2^{\alpha-1} \frac{\alpha}{1+\alpha} \varrho_{\min }^{\alpha+1}\|\tilde{\theta}\|^{\alpha+1}+\frac{2 p \alpha}{1+\alpha}\left(2^{\alpha-2}+\frac{\alpha^{\alpha-1}}{(1+\alpha)^{\alpha}}\right)\|C\|^{\alpha+1}\|\delta\|_{\infty}^{\alpha+1},
$$

or

$$
\|\tilde{\theta}\| \geq \frac{p^{\frac{1}{\alpha+1}}\left[(1+\alpha)^{2}+2^{2-\alpha} \alpha^{\alpha-1}\right]^{\frac{1}{\alpha+1}}}{\varrho_{\min }(1+\alpha)^{\frac{\alpha}{\alpha+1}}}\|C\|\|\delta\|_{\infty} \Longrightarrow \dot{V}_{\tilde{\theta}} \leq-\frac{2^{\alpha-1} \alpha}{1+\alpha} \varrho_{\min }^{\alpha+1}\|\tilde{\theta}\|^{\alpha+1}
$$


Thus, from (34) and (32), it follows that

$$
\dot{V}_{\tilde{\theta}} \leq-\left(\frac{2^{\alpha-1} \alpha \varrho_{\min }^{\alpha+1} c_{2}^{\frac{\alpha+1}{2}}}{1+\alpha}\right) V_{\tilde{\theta}}^{\frac{\alpha+1}{2}}, \forall\|\tilde{\theta}\| \geq \frac{p^{\frac{1}{\alpha+1}}\left[(1+\alpha)^{2}+2^{2-\alpha} \alpha^{\alpha-1}\right]^{\frac{1}{\alpha+1}}}{\varrho_{\min }(1+\alpha)^{\frac{\alpha}{\alpha+1}}}\|C\|\|\delta\|_{\infty} .
$$

Consequently, based on Lemma 6, $V_{\tilde{\theta}}$ is an ISS Lyapunov function for system (13), and the system (13) with $\alpha \in[0,1)$ is $I S S$ with respect to the input $\delta$, and hence its trajectories also satisfy the bounds given by (15) and (16) for any $\alpha \in[0,1)$.

Proof of Lemma 4: Consider the candidate Lyapunov function

$$
V_{\delta}=\delta^{T} P \delta
$$

which satisfies the following inequality

$$
\lambda_{\min }(P)\|\delta\|^{2} \leq V_{\delta} \leq \lambda_{\max }(P)\|\delta\|^{2} .
$$

The time derivative of $V_{\delta}$ along the trajectories of (14) is given by

$$
\dot{V}_{\delta}=\delta^{T}\left[(A-L C)^{T} P+P(A-L C)\right] \delta+2 \delta^{T} P D(w-k \operatorname{sign}[F(y-C \hat{x})]),
$$

then, if (17)-(18) are satisfied it follows that

$$
\begin{gathered}
\dot{V}_{\delta} \leq-\delta^{T}\left[\beta^{-1} P+(\beta r+2 \varpi) C^{T} C\right] \delta+2 \delta^{T} C^{T} F^{T}(w-k \operatorname{sign}[F(y-C \hat{x})]), \\
\leq-\delta^{T}\left[\beta^{-1} P+(\beta r+2 \varpi) C^{T} C\right] \delta+2(y-C \hat{x})^{T} F^{T}(w-k \operatorname{sign}[F(y-C \hat{x})]) \\
\quad-2 \tilde{\theta}^{T} \Omega^{T} C^{T} F^{T}(w-k \operatorname{sign}[F(y-C \hat{x})]), \\
\leq-\delta^{T}\left[\beta^{-1} P+(\beta r+2 \varpi) C^{T} C\right] \delta+2\|F(y-C \hat{x})\|\|w\|_{\infty}-2 k\|F(y-C \hat{x})\| \\
-2 \tilde{\theta}^{T} \Omega^{T} C^{T} F^{T}(w-k \operatorname{sign}[F(y-C \hat{x})]),
\end{gathered}
$$

and, if one fixes $k=\|w\|_{\infty}$ it is given that

$$
\begin{aligned}
\dot{V}_{\delta} & \leq-\delta^{T}\left[\beta^{-1} P+(\beta r+2 \varpi) C^{T} C\right] \delta+2\|C \Omega \tilde{\theta}\|\|F\|\|w\|_{\infty}(1+\|\operatorname{sign}[F(y-C \hat{x})]\|), \\
& \leq-\delta^{T}\left[\beta^{-1} P+(\beta r+2 \varpi) C^{T} C\right] \delta+4\|C \Omega \tilde{\theta}\|\|F\|\|w\|_{\infty}, \\
& \leq-\delta^{T}\left(\beta^{-1} P+\varpi C^{T} C\right) \delta-(\beta r+\varpi) \delta^{T} C^{T} C \delta+4\|C \Omega \tilde{\theta}\|\|F\|\|w\|_{\infty} .
\end{aligned}
$$

Recalling that $C \delta=y-C \hat{x}+C \Omega \tilde{\theta}$, then

$$
\begin{gathered}
\dot{V}_{\delta} \leq-\delta^{T}\left(\beta^{-1} P+\varpi C^{T} C\right) \delta-(\beta r+\varpi)(y-C \hat{x}+C \Omega \tilde{\theta})^{T}(y-C \hat{x}+C \Omega \tilde{\theta}) \\
\quad+4\|C \Omega \tilde{\theta}\|\|F\|\|w\|_{\infty} \\
\leq-\delta^{T}\left(\beta^{-1} P+\varpi C^{T} C\right) \delta-(\beta r+\varpi)\|y-C \hat{x}\|^{2}-(\beta r+\varpi)\|C \Omega \tilde{\theta}\|^{2} \\
\quad+2(\beta r+\varpi)\|y-C \hat{x}\|\|C \Omega \tilde{\theta}\|+4\|C \Omega \tilde{\theta}\|\|F\|\|w\|_{\infty} .
\end{gathered}
$$

Using the fact that

$$
\begin{gathered}
-\varpi\|C \Omega \tilde{\theta}\|^{2}+4\|C \Omega \tilde{\theta}\|\|F\|\|w\|_{\infty} \leq 4 \varpi^{-1}\|F\|^{2}\|w\|_{\infty}^{2}, \\
-(\beta r+\varpi)\|y-C \hat{x}\|^{2}+2(\beta r+\varpi)\|y-C \hat{x}\|\|C \Omega \tilde{\theta}\| \leq(\beta r+\varpi)\|C \Omega \tilde{\theta}\|^{2},
\end{gathered}
$$

it is obtained

$$
\begin{aligned}
\dot{V}_{\delta} & \leq-\delta^{T}\left(\beta^{-1} P+\varpi C^{T} C\right) \delta-\beta r\|C \Omega \tilde{\theta}\|^{2}+(\beta r+\varpi)\|C \Omega \tilde{\theta}\|^{2}+4 \varpi^{-1}\|F\|^{2}\|w\|_{\infty}^{2} \\
& \leq-\delta^{T}\left(\beta^{-1} P+\varpi C^{T} C\right) \delta+\varpi\|C \Omega \tilde{\theta}\|^{2}+4 \varpi^{-1}\|F\|^{2}\|w\|_{\infty}^{2} \\
& \leq-\alpha_{\delta}\|\delta\|^{2}+\varpi\|C \Omega \tilde{\theta}\|^{2}+4 \varpi^{-1}\|F\|^{2}\|w\|_{\infty}^{2}
\end{aligned}
$$


where $\alpha_{\delta}=\beta^{-1} \lambda_{\min }(P)+\varpi\|C\|^{2}$. Then, for all $\rho \in(0,1)$, it follows that

$$
\dot{V}_{\delta} \leq-(1-\rho) \alpha_{\delta}\|\delta\|^{2}, \forall\|\delta\| \geq \sqrt{\frac{\varpi \varrho_{\max }^{2}}{\rho \alpha_{\delta}}}\|\tilde{\theta}\|_{\infty}+\frac{2\|F\|\|w\|_{\infty}}{\sqrt{\rho \alpha_{\delta}}}
$$

Then, from (37) and (36), it is given that

$$
\dot{V}_{\delta} \leq-\left(\frac{(1-\rho) \alpha_{\delta}}{\lambda_{\max }(P)}\right) V_{\delta}, \forall\|\delta\| \geq \sqrt{\frac{\varpi \varrho_{\max }^{2}}{\rho \alpha_{\delta}}}\|\tilde{\theta}\|_{\infty}+\frac{2\|F\|\|w\|_{\infty}}{\sqrt{\rho \alpha_{\delta}}} .
$$

Therefore, based on Lemma $6, V_{\delta}$ is an ISS Lyapunov function for system (14), and the system (14) is ISS with respect to the inputs $\tilde{\theta}$ and $w$, and hence its trajectories satisfy the bounds given by (19) and (20).

Proof of Theorem 5: Let us consider the following functions

$$
V_{\tilde{\theta}}=\frac{1}{2} \tilde{\theta}^{T} \Gamma^{-1} \tilde{\theta}, V_{\delta}=\delta^{T} P \delta .
$$

Based on Lemmas 3 and 4, one can conclude that

$$
\begin{gathered}
\left(2 \lambda_{\max }(\Gamma)\right)^{-1}\|\tilde{\theta}\|^{2} \leq V_{\tilde{\theta}} \leq\left(2 \lambda_{\min }(\Gamma)\right)^{-1}\|\tilde{\theta}\|^{2}, \\
V_{\tilde{\theta}} \geq\left(\frac{p^{\frac{2}{\alpha+1}}\left[(1+\alpha)^{2}+2^{2-\alpha} \alpha^{\alpha-1}\right]^{\frac{2}{\alpha+1}}\|C\|^{2}}{2 \varrho_{\min }^{2}(1+\alpha)^{\frac{2 \alpha}{\alpha+1}} \lambda_{\max }(P) \lambda_{\max }(\Gamma)}\right) V_{\delta}, \\
\Rightarrow \dot{V}_{\tilde{\theta}} \leq-\left(\frac{2^{\alpha-1} \alpha \varrho_{\min }^{\alpha+1}\left(2 \lambda_{\min }(\Gamma)\right)^{\frac{\alpha+1}{2}}}{1+\alpha}\right) V_{\tilde{\theta}}^{\frac{\alpha+1}{2}},
\end{gathered}
$$

and

$$
\begin{gathered}
\lambda_{\min }(P)\|\delta\|^{2} \leq V_{\delta} \leq \lambda_{\max }(P)\|\delta\|^{2}, \\
V_{\delta} \geq \max \left[\left(\sqrt{\frac{4 \beta \varpi \varrho_{\max }^{2} \lambda_{\min }^{2}(\Gamma)}{\rho \lambda_{\min }(P)}}\right) V_{\tilde{\theta}},\left(\frac{2\|F\|}{\sqrt{\rho} \varpi\|C\|}\right)\|w\|\right] \\
\Rightarrow \dot{V}_{\delta} \leq-\left(\frac{(1-\rho) \alpha_{\delta}}{\lambda_{\max }(P)}\right) V_{\delta} .
\end{gathered}
$$

Therefore, the conditions (6)-(8) are satisfied with

$$
\begin{gathered}
\psi_{11}(\|\tilde{\theta}\|)=\left(2 \lambda_{\max }(\Gamma)\right)^{-1}\|\tilde{\theta}\|^{2}, \psi_{12}(\|\tilde{\theta}\|)=\left(2 \lambda_{\min }(\Gamma)\right)^{-1}\|\tilde{\theta}\|^{2}, \\
\psi_{13}\left(V_{\tilde{\theta}}\right)=\left(\frac{2^{\alpha-1} \alpha \varrho_{\min }^{\alpha+1}\left(2 \lambda_{\min }(\Gamma)\right)^{\frac{\alpha+1}{2}}}{1+\alpha}\right) V_{\tilde{\theta}}^{\frac{\alpha+1}{2}} \\
\psi_{21}(\|\delta\|)=\lambda_{\min }(P)\|\delta\|^{2}, \psi_{22}(\|\delta\|)=\lambda_{\max }(P)\|\delta\|^{2}, \psi_{23}\left(V_{\delta}\right)=\left(\frac{(1-\rho) \alpha_{\delta}}{\lambda_{\max }(P)}\right) V_{\delta}, \\
\chi_{1}\left(V_{\delta}(\delta)\right)=\left(\frac{p^{\frac{2}{\alpha+1}}\left[(1+\alpha)^{2}+2^{2-\alpha} \alpha^{\alpha-1}\right]^{\frac{2}{\alpha+1}}\|C\|^{2}}{2 \varrho_{\min }^{2}(1+\alpha)^{\frac{2 \alpha}{\alpha+1}} \lambda_{\max }(P) \lambda_{\max }(\Gamma)}\right) V_{\delta}, \\
\gamma_{1}(\|w\|)=0, \kappa_{1}=0, \\
\chi_{2}\left(V_{\tilde{\theta}}(\tilde{\theta})\right)=\left(\sqrt{\frac{4 \beta \varpi \varrho_{\max }^{2} \lambda_{\min }^{2}(\Gamma)}{\rho \lambda_{\min }(P)}}\right) V_{\tilde{\theta}} \\
\gamma_{2}(\|w\|)=\left(\frac{2\|F\|}{\sqrt{\rho} \varpi\|C\|}\right)\|w\|, \kappa_{2}=0
\end{gathered}
$$

and then, it follows that

$$
\chi_{1} \circ \chi_{2}(\mathrm{r})=\left(\frac{p^{\frac{2}{\alpha+1}}\left[(1+\alpha)^{2}+2^{2-\alpha} \alpha^{\alpha-1}\right]^{\frac{2}{\alpha+1}}\|C\|^{2} \sqrt{\beta \varpi} \varrho_{\max } \lambda_{\min }(\Gamma)}{\varrho_{\min }^{2}(1+\alpha)^{\frac{2 \alpha}{\alpha+1}} \lambda_{\max }(P) \lambda_{\max }(\Gamma) \sqrt{\rho \lambda_{\min }(P)}}\right) \mathrm{r} .
$$


Hence, if the parameters $P, \Gamma, \alpha, \beta, \varpi$ and $\rho$ are such that the matrix inequality (21) holds, then one obtains that

$$
\chi_{1} \circ \chi_{2}(\mathrm{r})<r, \forall r>0 .
$$

Therefore, all the conditions of Theorem 2 are satisfied, and thus, one concludes that the interconnected error system (13)-(14) is $I S S$ with respect to the external input $w$ for any $\alpha \in[0,1)$. Moreover, with $w=0$, the system (13)-(14) is globally asymptotically stable.

\section{REFERENCES}

1. Ioannou PA, Sun J. Robust Adaptive Control. Prentice Hall, Inc.: New Jersey, USA, 1996.

2. Astolfi A, Karagiannis D, Ortega R. Nonlinear and Adaptive Control with Applications. Communications and Control Engineering, Springer-Verlag: London, 2008.

3. Besançon G. Nonlinear Observers and Application. Springer-Verlang Berlin Heidelberg, 2007.

4. Sastry S, Bodson M. Adaptive Control. Stability, Convergence and Robustness. Prentice Hall Advance Reference Series: New Jersey, USA, 1989.

5. Lüders G, Narendra K. An adaptive observer and identifier for linear systems. IEEE Transactions on Automatic Control 1973; 18(5):496-499.

6. Carroll R, Lindorff D. An adaptive observer for single input single output linear systems. IEEE Transactions on Automatic Control 1973; 18(5):428-435.

7. Narendra K, Annaswamy A. Stable Adaptive Systems. Dover Publications: Mineola, NY, 2005.

8. Bastin G, Gevers M. Stable adaptive observers for nonlinear time-varying systems. IEEE Transactions on Automatic Control 1988; 33(7):650-658.

9. Marino R, Tomei P. Global adaptive observers for nonlinear systems via filteres transformations. IEEE Transactions on Automatic Control 1992; 37(8):1239-1245.

10. Marino R, Tomei P. Nonlinear Control Design: Geometric, Adaptive \& Robust. London, Prentice Hall, 1995.

11. Besançon G. Remarks on nonlinear adaptive observer design. Systems \& Control Letters 2000; 41:271-280.

12. Zhang Q. Adaptive observer for multiple-input-multiple-output (MIMO) linear time varying systems. IEEE Transactions on Automatic Control March 2002; 47(3):525-529.

13. Xu A, Zhang Q. Nonlinear system fault diagnosis based on adaptive estimation. Automatica 2004; 40(7):11811193.

14. Farza M, M'Saad M, Maatoug T, Kamoun M. Adaptive observers for nonlinearly parameterized class of nonlinear systems. Automatica 2009; 45:2292-2299.

15. Liu Y. Robust adaptive observer for nonlinear systems with unmodeled dynamic. Automatica 2009; 45:1891-1895.

16. Stamnes O, Aamo O, Kaasa GO. Redesign of adaptive observers for improved parameter identification in nonlinear systems. Automatica 2011; 47:403-410.

17. Tyukin IY, Steur E, Nijmeijer H, van Leeuwen C. Adaptive observers and parameter estimation for a class of systems nonlinear in the parameters. Automatica 2013; 49:2409-2423.

18. Chen WH, Yang W, Zheng WX. Adaptive impulsive observers for nonlinear systems: Revisited. Automatica 2015; 61:232-240.

19. Efimov D, Fradkov A. Design of impulsive adaptive observers for improvement of persistency of excitation. International Journal of Adaptive Control and Signal Processing 2015; 29(6):756-782.

20. Folin T, Ahmed-Ali T, Giri F, Burlion L, Lamnabhi-Lagarrigue F. Sampled-data adaptive observer for a class of state-affine output-injection nonlinear systems. IEEE Transactions on Automatic Control 2016; 61(2):462-467.

21. Ahmed-Ali T, Giri F, Krstic M, Burlion L, Lamnabhi-Lagarrigue F. Adaptive observer design with heat pde sensor. Automatica 2017; 82:93-100.

22. Shtessel Y, Edwards C, Fridman L, Levant A. Sliding Mode Control and Observation. Birkhauser: New York, 2014.

23. Yan XG, Edwards C. Adaptive sliding-mode-observer-based fault reconstruction for nonlineary systems with parametric uncertainties. IEEE Transactions on Industrial Electronics November 2008; 55(11):4029-4037.

24. Efimov D, Edwards C, Zolghadri A. Enhancement of adaptive observer robustness applying sliding mode techniques. Automatica 2016; 72:53-56.

25. Jiang ZP, Mareels I, Wang Y. A Lyapunov formulation of the nonlinear small-gain theorem for interconnected ISS systems. Automatica 1996; 32(8):1211-1215.

26. Dashkovskiy S, Efimov D, Sontag E. Input to state estability and allied system properties. Automation and Remote Control 2011; 72(8):1579-1614.

27. Bernuau E, Polyakov A, Efimov D, Perruquetti W. Verification of ISS, iISS and IOSS properties applying weighted homogeneity. Systems \& Control Letters 2013; 62(12):1159-1167.

28. Efimov D, Fradkov A. Adaptive tunning to bifurcation for time-varying nonlinear systems. Automatica 2006; 42(3):417-425.

29. Edwards C, Yan XG, Spurgeon S. On the solvability of the constrained Lyapunov problem. IEEE Transactions on Automatic Control 2007; 52(10).

30. Poznyak A. Advanced Mathematical Tools for Control Engineers: Volume 1: Deterministic Techniques. Elsevier Science: Amsterdam-Boston, 2008.

31. Khalil H. Nonlinear Systems. Prentice Hall: New Jersey, U.S.A., 2002. 